\title{
Kinetics and Pathways of the Aqueous Photolysis of Pharmaceutical Pollutants: A Versatile Laboratory or Remote Learning Investigation
}

\section{Journal Article}

Author(s):

Ossola, Rachele (1D; Partanen, Sarah B.; McNeill, Kristopher (1); Arnold, William A.; O'Connor, Meghan; Latch, Douglas E.; Buth, Jeffrey M.

Publication date:

2021-07-13

Permanent link:

https://doi.org/10.3929/ethz-b-000498261

Rights / license:

Creative Commons Attribution-NonCommercial-NoDerivatives 4.0 International

Originally published in:

Journal of Chemical Education 98(7), https://doi.org/10.1021/acs.jchemed.0c01398

Funding acknowledgement:

188565 - Reactivity-based characterization of photoexcited natural organic matter (SNF) 


\title{
Kinetics and Pathways of the Aqueous Photolysis of Pharmaceutical Pollutants: A Versatile Laboratory or Remote Learning Investigation
}

\author{
Jeffrey M. Buth,* Rachele Ossola, Sarah B. Partanen, Kristopher McNeill, William A. Arnold, \\ Meghan O’Connor, and Douglas E. Latch*
}

Cite This: J. Chem. Educ. 2021, 98, 2411-2418

Read Online

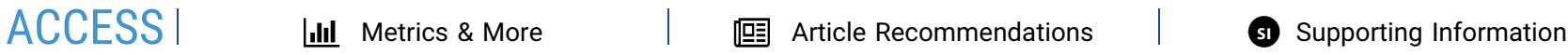

ABSTRACT: In this laboratory experiment, students explore the aquatic photochemical fate of ranitidine and cimetidine, two common pharmaceutical pollutants found in wastewater. It provides an engaging environmental context for students to develop knowledge of reaction kinetics and photochemistry as well as skill in using analytical instrumentation. This versatile experiment consists of two basic modules, three optional advanced modules, and additional add-ons that may be performed in various combinations to meet the unique learning objectives of general, analytical, physical, and environmental chemistry courses and science outreach activities. It may be performed as a traditional lab
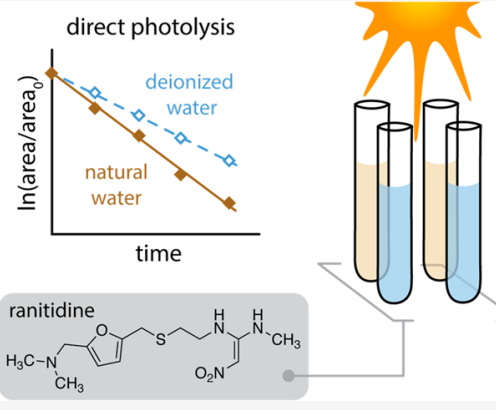
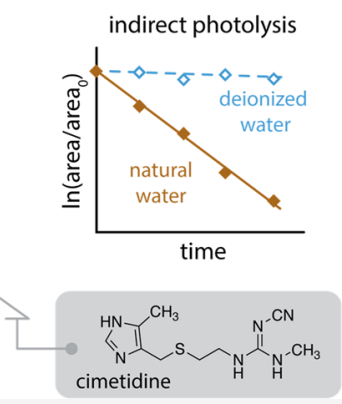
experiment or as an entirely remote exercise with an increased focus on data analysis and interpretation using provided example data sets. All of the photolysis experiments are carried out by preparing solutions of ranitidine or cimetidine in various matrices, irradiating the samples, and periodically removing subsamples for HPLC analysis of the compound of interest. Pseudo-first order kinetic plots are then generated to determine rate constants that are used to draw conclusions about photolysis pathways or to calculate additional kinetic parameters. In the two basic modules, cimetidine is found to degrade appreciably only when irradiated in the presence natural organic matter (NOM), indicating an indirect, photosensitized degradation pathway. In contrast, ranitidine degrades in pure buffer and in the presence of NOM with comparable rate constants, highlighting the predominant role of direct photolysis. In the advanced modules, students calculate ranitidine direct photolysis quantum yields and examine the significance of singlet oxygen as a photochemically produced reactive intermediate. The two basic modules may be completed in two 3 hour lab periods, while the advanced modules require additional time. This experiment requires only an HPLC instrument, inexpensive chemicals, and common glassware and lab equipment if performed in person and a personal computer if performed remotely.

KEYWORDS: First-Year Undergraduate/General, Second-Year Undergraduate, Upper-Division Undergraduate, Analytical Chemistry, Environmental Chemistry, Laboratory Instruction, Physical Chemistry, Distance Learning/Self Instruction, Drugs/Pharmaceuticals, Kinetics, Photochemistry

\section{INTRODUCTION}

The chemical education community has long embraced the need for laboratory experiments that engage students in societally important contexts of personal interest rather than those that explore isolated topics without a practical purpose. $^{1,2}$ The natural environment was identified as a context for undergraduate experiments that facilitates meaningful investigations, realistic problem solving, and promotion of teamwork. ${ }^{3}$ While recognizing the value of context-based learning, Sevian and Talanquer cautioned that in some cases a focus on the contexts that stimulate student engagement may take precedence over the learning of core chemistry ideas and practices. ${ }^{4}$ Several recent reports of laboratory experiments have demonstrated that investigating the reactivity of environmentally significant chemicals by natural and engineered processes such as hydrolysis, ${ }^{5}$ oxidation, ${ }^{6}$ dissolution, ${ }^{7}$ and catalyzed reduction ${ }^{8}$ provides a stimulating context while developing an understanding of fundamental concepts and skill in analytical techniques.

Pharmaceuticals in particular have garnered much attention as aquatic pollutants over the last two decades. ${ }^{9,10}$ Pharmaceuticals present in the discharge from wastewater treatment plants and the runoff from livestock production facilities to surface waters threaten the health of aquatic ecosystems because of their potential to elicit potent biological

Received: November 16, 2020

Revised: $\quad$ May 6, 2021

Published: June 22, 2021 
effects at low concentrations, including the proliferation of antibiotic resistance, endocrine system disruption, and acute toxicity. ${ }^{11,12}$ Communities that derive their potable water from contaminated sources may also be affected. ${ }^{13}$ Solar photolysis has been established as a potential pathway by which many pharmaceuticals and other pollutants are degraded in natural waters. ${ }^{14,15}$ While several undergraduate lab experiments have explored photochemistry in various settings (e.g., gas-phase photolysis, ${ }^{16}$ organic synthesis, ${ }^{17}$ and photopolymerization ${ }^{18}$ ), we are unaware of any that have investigated the aqueous photolysis of environmental pollutants.

We have developed an experiment that explores aquatic photochemistry while focusing on an important pollutant class with which students have everyday familiarity. This laboratory experiment compares the environmental photoreactivity of the over-the-counter antacid drugs ranitidine and cimetidine, sold under the trade names Zantac and Tagamet, respectively. (Zantac was recently pulled from the market because of concerns about contamination by the carcinogen nitrosodimethylamine). ${ }^{19}$ These drugs photodegrade via different pathways. Ranitidine reacts predominately via direct photolysis. $^{20}$ It absorbs solar radiation $(h \nu)$, producing an excited state that subsequently degrades:

$$
\text { ranitidine }+h \nu \rightarrow \text { ranitidine* } \rightarrow \text { photoproducts }
$$

The reaction is thus first-order with respect to ranitidine. The efficiency of this degradation pathway is quantified as the direct photolysis quantum yield $\left(\Phi_{\text {dir }}\right)$, defined as moles of ranitidine degraded per moles of photons absorbed.

In contrast, cimetidine does not absorb light within the solar spectrum but instead photolyzes indirectly primarily via reaction with photochemically generated singlet oxygen $\left({ }^{1} \mathrm{O}_{2}\right) .{ }^{20} \mathrm{In}$ aquatic environments, ${ }^{1} \mathrm{O}_{2}$ is formed upon energy transfer from the triplet excited states of natural organic matter $\left({ }^{3} \mathrm{NOM}^{*}\right)$ to ground-state oxygen. ${ }^{21}$ Cimetidine then reacts with ${ }^{1} \mathrm{O}_{2}$ to form degradation products:

$$
\begin{aligned}
& \mathrm{NOM}+h \nu \rightarrow{ }^{3} \mathrm{NOM}^{*} \\
& { }^{3} \mathrm{NOM}^{*}+\mathrm{O}_{2} \rightarrow \mathrm{NOM}+{ }^{1} \mathrm{O}_{2} \\
& { }^{1} \mathrm{O}_{2}+\text { cimetidine } \rightarrow \text { products }
\end{aligned}
$$

Typically, a steady-state concentration of ${ }^{1} \mathrm{O}_{2}$ is maintained in surface waters under steady irradiation. The rate of ${ }^{1} \mathrm{O}_{2}$ mediated loss of cimetidine is thus pseudo-first order with respect to cimetidine and depends on the ${ }^{1} \mathrm{O}_{2}$ steady-state concentration. ${ }^{20}$ The intrinsic reactivity of cimetidine with ${ }^{1} \mathrm{O}_{2}$ is quantified by its second-order rate constant $\left(k_{\mathrm{rxn},}^{1} \mathrm{O}_{2}\right)$.

This investigation has been designed for students to develop and apply several core concepts and skills in an integrated and authentic context. It may be readily adapted to accomplish specific pedagogical goals appropriate to various levels of the undergraduate curriculum in introductory, analytical, physical, and environmental chemistry courses. The investigation may also be performed remotely with a focus on data analysis and interpretation. From this experiment, students will learn to:

(1) Appreciate the unintended environmental impacts of chemicals used in everyday life. This learning objective provides the authentic context that drives student engagement.

(2) Differentiate between direct and indirect photochemistry pathways. This learning goal applies to physical and environmental chemistry students.
(3) Analyze kinetic data to quantify parameters such as rate constants, half-lives, and quantum yields. This is an important learning goal for general, environmental, and physical chemistry courses.

(4) Perform analyses using modern analytical instrumentation (UV-vis spectroscopy and high-pressure liquid chromatography (HPLC)). This learning goal applies to several course types.

(5) Perform experiments with appropriate controls and interpret the resultant data to systematically determine how factors such as the environmental matrix affect a chemical's reactivity. As many undergraduates lack the conceptual understanding to design effective experimental controls, ${ }^{22}$ this learning goal addresses a need across the undergraduate chemistry curriculum.

\section{EXPERIMENT DESCRIPTION}

The experiment consists of a basic form (modules 1 and 2) and optional advanced modules (3-5) and add-ons (Figure 1), each module examining a different aspect of the direct or indirect photochemistry of ranitidine and/or cimetidine. Instructors may select subsets of the five modules and addons on the basis of the unique learning objectives of their courses or outreach activities and the lab time available. Figure 1 highlights the course type for which each module is best-suited. Recommended module combinations along with the approximate amount of time each combination requires are supplied in the student instructions. In most circumstances, basic modules 1 and 2 may be completed in two 3 hour lab periods, while the completion of additional modules and addons may require additional time. Each module may be completed in the laboratory or remotely. Example raw data sets for each module are supplied in an Excel spreadsheet containing spectral and kinetic data sets for the remote investigation. Corresponding fully analyzed data sets are additionally provided in this document.

The experimental modules have been completed in a variety of chemistry courses at various types of institutions. All five modules have been taught once as an in-person laboratory experiment and once as a remote learning experience in an upper-division environmental chemistry course of 16-18 students at a large research university. Students worked in groups of three to four in person and individually in the remote version. Modules 1 and 2 have been conducted in-person eight times in an upper-division instrumental analysis course of approximately 8-20 students per section at a doctoral/ professional university with undergraduate-only instruction in the physical sciences and once in a lower-division environmental chemistry course of 13 students at a small, primarily undergraduate university. In these two courses, students worked in teams of two (preferred) to four depending on the class size. A simplified version of modules 1 and 2 was conducted in-person three times in a high school outreach program with cohorts of approximately 20 students working in groups of four.

A procedural overview of the basic and advanced modules is provided below; further details are supplied in the student instructions and instructor prep notes. All of the photolyses may be conducted under sunlight or an alternative light source, though for consistency the same light source should be used for all modules. Light source options are described in greater depth on page S3 in the instructor prep notes. As shown in 

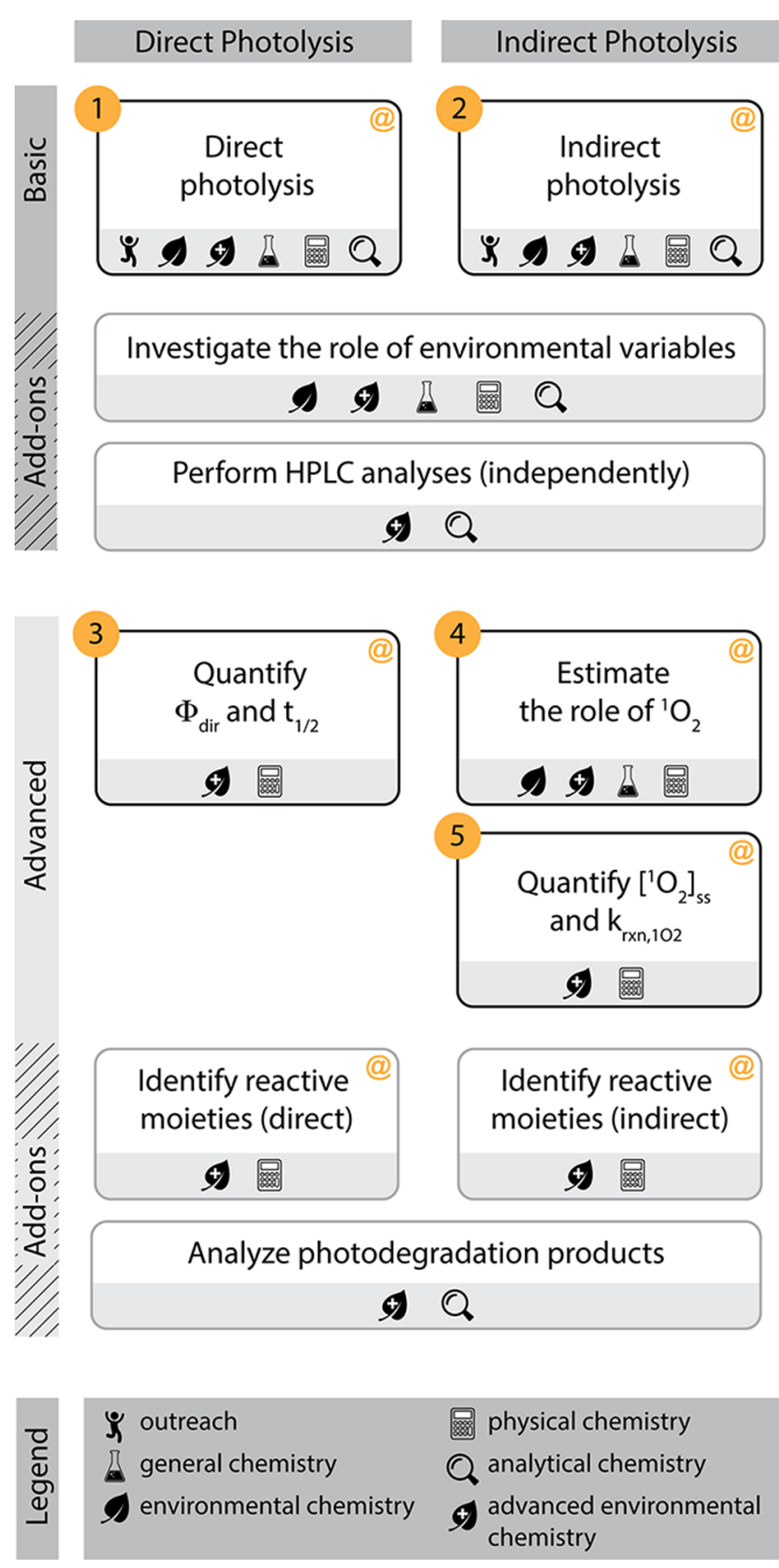

Figure 1. Schematic overview of the basic modules (1 and 2) and the optional advanced modules (3-5). It is anticipated that in most cases basic modules 1 and 2 will be completed, with optional advanced modules and add-ons chosen on the basis of the learning objectives of a particular course. Pictograms indicate the course type for which each module and add-on is best suited, and the @ symbol indicates those for which sample data sets are provided for remote learning.
Figure 2, photolysis experiments are performed by preparing drug solutions in various matrices, irradiating samples, periodically removing subsamples, and analyzing them by HPLC for the compound of interest. Pseudo-first order kinetic graphs are constructed by plotting the natural logarithm of the ratio of a compound's HPLC peak area at each time point to its initial peak area versus time. The pseudo-first order photolysis rate constant is obtained from the absolute value of the slope. In each module, a dark control of ranitidine and/or cimetidine wrapped in foil is placed alongside the experimental samples during irradiation to confirm that any observed degradation in the light-exposed samples was the result of photolysis and not another process (e.g., thermal degradation, hydrolysis, etc.).

Basic Modules 1 and 2: Determination of Photolysis Rate Constants in Phosphate Buffer and Natural Water

The purpose of module 1 is to determine the first-order rate constants for ranitidine and cimetidine in phosphate buffer, where direct photolysis is the only operative photodegradation pathway. Module 2 aims to determine pseudo-first order rate constants for the two compounds in filtered natural water, where indirect photolysis may occur in addition to direct photolysis. Modules 1 and 2 have typically been conducted together over two 3 hour lab periods to compare the relative significance of direct and indirect photolysis for each compound. In the first period, students prepare the buffer, obtain and filter natural water, make $\mathrm{pH}$ measurements, prepare drug solutions, and record UV-vis absorption spectra of ranitidine and cimetidine. In the second period, students perform the photolyses, and HPLC analyses are conducted by the instructor.

It is possible to complete modules 1 and 2 in a single 3 hour lab period by preparing filtered natural water and drug solutions and recording UV-vis absorption spectra in advance. For additional time efficiency, especially if an HPLC autosampler is not available, some students may be assigned to complete only module 1 while others complete module 2 . Likewise, some students may be assigned to complete a module for ranitidine only while others do so for cimetidine. The raw data from each group may then be pooled and analyzed individually at the end.

\section{Add-Ons to Modules 1 and 2}

In carrying out modules 1 and 2 , students in particular courses may benefit from optional add-on components, as denoted in Figure 1. First, in addition to comparing photolysis rates for the drugs in phosphate buffer and natural water, students may investigate the effect of other environmental variables on the photolysis rate of one or both compounds. In a lower-division environmental chemistry course, after completing modules 1 and 2 as described above, students explored the effect of a variable such as $\mathrm{pH}$, ionic strength, or natural water source on

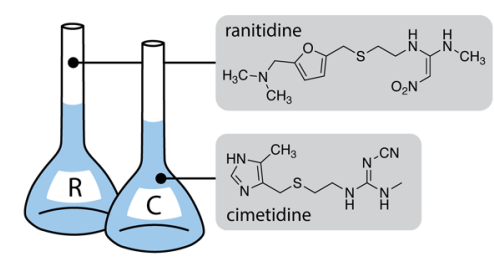

1. Prepare solutions

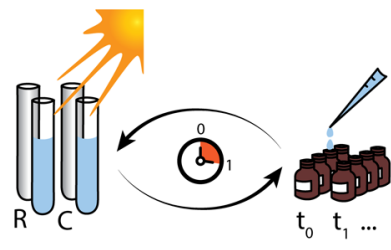

2. Irradiate and take time points

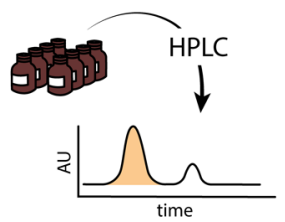

3. Analyze the samples

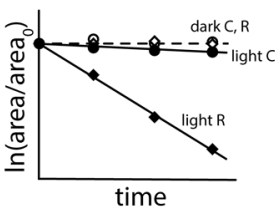

4. Analyze the data

Figure 2. General sequence of steps to be completed in each photolysis experiment. 
the photolysis rate of one of the compounds over two additional 3 hour lab periods. This provided an opportunity for inquiry-based learning and student-led experimental design. In an upper-division instrumental analysis course, students systematically studied how the NOM concentration affected the photolysis kinetics of cimetidine.

A second optional add-on to modules 1 and 2 is for students to perform the HPLC analyses to gain valuable hands-on experience with operating analytical instrumentation. In an upper-division analytical chemistry course, an instructor or teaching assistant guided student teams in preparing the mobile phase, priming the HPLC system, attaching and flushing the column, creating a method, and running a sample sequence.

Advanced Module 3: Determination of the Direct Photolysis Quantum Yield and Environmental Half-Lives of Ranitidine

The purpose of module 3 is to measure the direct photolysis quantum yield of ranitidine and to estimate direct photolysis half-lives under environmentally relevant conditions. To measure the quantum yield, a solution of ranitidine in phosphate buffer is irradiated alongside a $p$-nitroanisole (PNA)/pyridine chemical actinometer solution, which is required to quantify the light intensity. ${ }^{23,24}$ While this module may be run independently in a dedicated lab period, it is most efficient to irradiate the PNA/pyridine actinometer solution alongside the solution of ranitidine in phosphate buffer in module 1. The UV-vis absorption spectrum of ranitidine from module 1 is used to determine ranitidine molar extinction coefficients for the quantum yield calculations. The direct photolysis quantum yield for ranitidine is then used to estimate environmental half-lives using published solar spectra for winter and summer sunlight conditions. ${ }^{25}$

\section{Advanced Module 4: Determination of the Role of Singlet Oxygen as a Reactive Intermediate}

The objective of module 4 is to assess whether singlet oxygen is a relevant reactive intermediate in the indirect photolysis of ranitidine and cimetidine. This is done qualitatively by examining the effects of singlet oxygen chemical quenchers and enhancers on the photolysis rates of ranitidine and cimetidine in natural water. Module 4 is a more advanced alternative to module 2 .

Solutions of ranitidine and cimetidine are prepared in natural water, both unamended and with singlet oxygen chemical quenchers (sodium azide for cimetidine and 1,4diazabicyclo[2.2.2] octane (DABCO) for ranitidine) or enhancers (deuterated water for both compounds). ${ }^{20}$ (While sodium azide works well as a ${ }^{1} \mathrm{O}_{2}$ quencher with cimetidine, there are complicating photoreactions between ranitidine and sodium azide. Thus, DABCO is used as an alternative ${ }^{1} \mathrm{O}_{2}$ quencher with ranitidine.) Because ranitidine undergoes direct photolysis in addition to indirect photolysis, a direct photolysis control consisting of ranitidine in phosphate buffer should also be prepared. The solutions are irradiated and analyzed by HPLC as shown in Figure 2. The significance of singlet oxygen as a reactive intermediate is assessed by comparing the magnitudes of pseudo-first order indirect photolysis rate constants in the presence of singlet oxygen quenchers and enhancers to that of the unamended sample.
Advanced Module 5: Measurement of $k_{\mathrm{rxn},}{ }^{1} \mathrm{O}_{2}$ and $\left[{ }^{1} \mathrm{O}_{2}\right]_{\mathrm{ss}}$

The objective of module 5 is to estimate the fraction of photodegradation that is due to singlet oxygen under environmentally relevant conditions. To perform this calculation, one must determine the second-order rate constants for the reaction of ranitidine and cimetidine with singlet oxygen $\left(k_{\mathrm{rxn},{ }^{1} \mathrm{O}_{2}}^{\mathrm{Ran}}\right.$ and $k_{\mathrm{rxn},{ }^{1} \mathrm{O}_{2}}^{\mathrm{Cim}}$, respectively) and the steady-state concentration of singlet oxygen in the natural water $\left(\left[{ }^{1} \mathrm{O}_{2}\right]_{\mathrm{ss}}\right)$. Module 5 should be run as an addition to module 2 or 4 .

To obtain the second-order rate constants, students irradiate phosphate-buffered ranitidine and cimetidine solutions containing a water-soluble zinc porphyrin, a pure photochemical source of singlet oxygen. ${ }^{26}$ These solutions also contain furfuryl alcohol (FFA), a molecular probe for singlet oxygen. The second-order rate constant for reaction with ${ }^{1} \mathrm{O}_{2}$ is obtained by comparing the degradation rate of FFA, for which $k_{\mathrm{rxn},{ }^{1} \mathrm{O}_{2}}^{\mathrm{FFA}}$ is known, to that of the drug.

To measure $\left[{ }^{1} \mathrm{O}_{2}\right]_{\mathrm{ss}}$ in natural water, a solution of FFA in natural water must also be irradiated. For efficiency, FFA may be added to the natural water solutions of ranitidine and cimetidine from module 2 or 4 that do not contain chemical quenchers or enhancers. The $\left[{ }^{1} \mathrm{O}_{2}\right]_{\mathrm{ss}}$ in natural water is obtained from the FFA depletion kinetics.

Add-Ons to Advanced Modules 3-5

Several optional add-ons to modules 3-5 may be completed to meet additional learning objectives of particular courses. First, physical chemistry or upper-division environmental chemistry students may use model compounds representing potentially reactive functional groups of ranitidine (Scheme $\mathrm{S} 1$ in the student instructions) to gain further insights into the mechanisms of direct and indirect photolysis. Because of the high cost of the model compounds and the length of time required for additional photolyses, sample data are provided for this add-on to be conducted as a remote learning activity. Comparison of the direct photolysis rate constants in phosphate buffer for the model compounds to that for ranitidine reveals the nitroguanidine functionality as the site that triggers direct photolysis. For singlet oxygen reactivity, comparison of the second-order ${ }^{1} \mathrm{O}_{2}$ rate constants for the model compounds of ranitidine with that for their parent compound reveals the aminomethylfuran moiety as the most likely site of ${ }^{1} \mathrm{O}_{2}$ attack.

As an additional add-on, upper-division analytical and environmental chemistry students may perform mass spectrometry analyses of photolyzed solutions to identify the photoproducts, introducing students to the potential generation of transformation products with increased toxicity relative to the parent compounds. This add-on provides further experience with sophisticated analytical instrumentation and spectral analysis.

\section{Remote Learning Investigation}

When the investigation was performed remotely, the understanding of theoretical concepts and the development of data analysis and interpretation skills were emphasized. In this context, students were given a video tutorial for each module that provided both the theory behind the experiments and a practical description of how the experiment would be conducted in the lab. Sample raw data sets for each module containing HPLC peak areas, UV-vis absorption spectral data, and an irradiance spectrum of the light source were provided 
to students to analyze and interpret. As noted above, these data sets are supplied in their raw and fully analyzed form in the Excel spreadsheet containing spectral and kinetic data sets.

\section{HAZARDS}

As with all laboratory work, eye protection should be worn at all times. Gloves should be worn when handling natural water samples to protect from potentially hazardous microorganisms. If an artificial light source is used, it should be enclosed, and UV protective eyewear should be worn. If the photolysis is performed outside, care should be taken in transporting glassware to avoid breakage. Hazards of specific chemicals are described in the Supporting Information. Used chemicals must be collected in labeled waste containers and disposed according to local regulations and procedures.

\section{RESULTS}

\section{Basic Modules 1 and 2}

When ranitidine and cimetidine were photolyzed in deionized water and natural water, plots of $\ln \left(\mathrm{area}_{t} / \mathrm{area}_{0}\right)$ versus time yielded linear fits, as shown in the example student data in Figure 3A. These results indicated that the reactions were firstorder with respect to the drug concentration. ${ }^{27}$ From each linear plot, the observed first-order rate constant, $\left(k_{\mathrm{obs}}^{\mathrm{A}}\right)$, was obtained as the absolute value of the slope of the fitted line, and the half-life was obtained from eq 1:

$$
\text { half-life }=\frac{\ln 2}{k_{\mathrm{obs}}^{\mathrm{A}}}
$$

An example of student-obtained rate constants and half-lives is presented in Table 1, corresponding to the kinetic data plotted in Figure 3A. While the pseudo-first order rate constants and half-lives obtained by students varied depending on the light source used, the trends presented in Figure $3 \mathrm{~A}$ and Table 1 were consistently observed. No significant decrease in drug peak areas was observed in dark controls, indicating that all compound loss in light-exposed samples was the result of photochemical processes.

Cimetidine did not photolyze appreciably in deionized water, indicating that it did not undergo direct photolysis. Rather, it required the NOM present in natural water in order to undergo indirect photolysis. This conclusion was supported by comparing the absorbance spectrum of cimetidine to the solar irradiance spectrum (Figure 3B). Because cimetidine does not absorb light at wavelengths above approximately 250 $\mathrm{nm}$, it cannot absorb within the wavelength range of solar emission $(>300 \mathrm{~nm})$ and cannot directly photolyze. Conversely, ranitidine degraded appreciably in deionized water without the presence of NOM, consistent with a direct photolysis pathway. The overlap of the absorption spectrum of ranitidine with the solar irradiance spectrum from approximately 300 to $360 \mathrm{~nm}$ provides further evidence for a direct photodegradation pathway (Figure 3B). Ranitidine degraded with a slightly higher rate constant in natural water compared with deionized water because of NOM-mediated indirect photolysis in addition to direct photolysis.

\section{Advanced Module 3}

As with modules 1 and 2, all data analysis performed in the advanced modules relied on the determination of pseudo-first order rate constants from the slope of $\ln \left(\mathrm{area}_{t} / \mathrm{area}_{0}\right)$ versus time plots. These rate constants were then used to obtain other
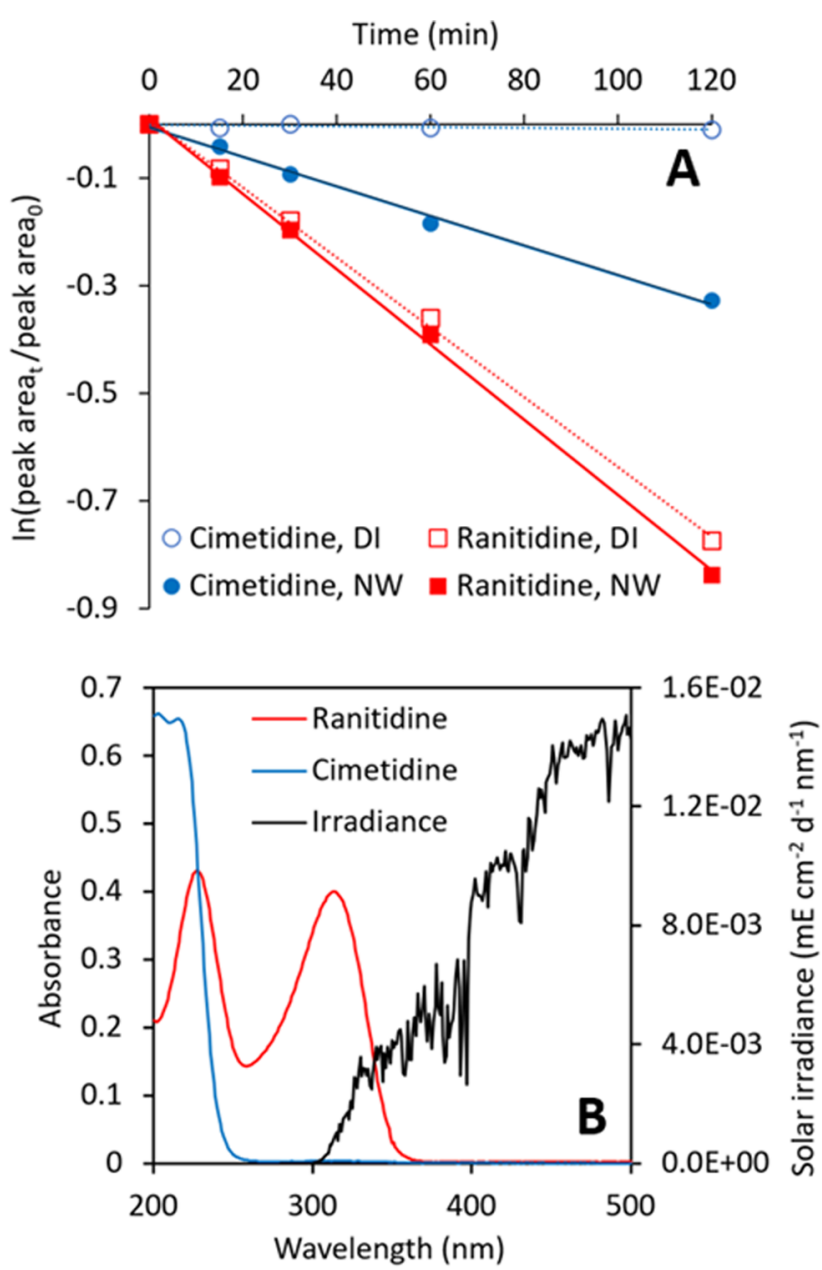

Figure 3. (A) Student-generated kinetic data for the photolysis of 50 $\mu \mathrm{M}$ ranitidine and cimetidine in deionized water (DI) and in filtered natural water (NW) under natural sunlight with no cloud cover (March 26, 2019, in Alliance, OH, USA). (B) Absorbance spectra of $25 \mu \mathrm{M}$ ranitidine and cimetidine at $\mathrm{pH} 7.3$ and daily average solar irradiance spectrum for March 20 at $40^{\circ} \mathrm{N}$ latitude (spectral data from ref 25).

Table 1. Student-Generated First-Order Rate Constants and Half-Lives Obtained for the Photolysis of Ranitidine and Cimetidine in Deionized Water (DI) and Natural Water (NW) Corresponding to the Data Plotted in Figure 3

$\begin{array}{lcc}\text { Compound (A) } & \text { Rate Constant } k_{\mathrm{obs}}^{\mathrm{A}}\left(\mathrm{h}^{-1}\right) & \text { Half-Life }(\mathrm{h}) \\ \text { Ranitidine, DI } & 3.89 \times 10^{-1} & 1.78 \\ \text { Ranitidine, NW } & 4.19 \times 10^{-1} & 1.66 \\ \text { Cimetidine, DI } & 4.31 \times 10^{-3} & 161 \\ \text { Cimetidine, NW } & 1.65 \times 10^{-1} & 4.20\end{array}$

parameters (calculation details are provided in the student instructions).

In module 3, the ranitidine direct photolysis quantum yield was calculated from the degradation rate constants of ranitidine and $p$-nitroanisole, the actinometer. An example student obtained a quantum yield value of $2.1 \times 10^{-3}$ (at $\mathrm{pH}$ 7.3 ), which is overall consistent with the literature. ${ }^{20}$ Because cimetidine does not undergo direct photolysis, direct photolysis quantum yields cannot be obtained.

From the direct photolysis quantum yield, molar extinction coefficients, and published sunlight spectra, $^{25}$ students can 
estimate the expected ranitidine half-life under environmentally relevant conditions. For example, one student used the quantum yield value above to calculate half-lives of 0.77 and $2.81 \mathrm{~h}$ for summer and winter noon light conditions, respectively, at $45^{\circ} \mathrm{N}$. Such a calculation can be a valuable addition to the remote learning experience, as students can better appreciate the effect of environmental variables on the fate of pollutants in the environment. For lower-division students, instructors may provide a spreadsheet containing computed rate constants for different environmental conditions and ask students to input their measured quantum yield and comment on the results. A fully worked out example of this calculation is provided in the Excel spreadsheet containing spectral and kinetic data sets.

\section{Advanced Module 4}

From the data for module 4, students qualitatively evaluated the results of the $\ln \left(\right.$ area $_{t} /$ area $\left._{0}\right)$ versus time plots to assess the effect of ${ }^{1} \mathrm{O}_{2}$ quenchers and enhancers on the ranitidine and cimetidine photodegradation kinetics. They correctly observed that ${ }^{1} \mathrm{O}_{2}$ reactivity is more important for cimetidine than for ranitidine on the basis of the magnitude of changes for the two compounds. For ranitidine, they also noted that direct photolysis still plays an important role in the presence of NOM.

\section{Advanced Module 5}

Data from module 5 were analyzed to yield second-order ${ }^{1} \mathrm{O}_{2}$ rate constants and steady-state concentrations of singlet oxygen in natural water. An example student obtained second-order rate constants of $2.6 \times 10^{7} \mathrm{M}^{-1} \mathrm{~s}^{-1}$ for ranitidine and $2.4 \times 10^{8} \mathrm{M}^{-1} \mathrm{~s}^{-1}$ for cimetidine (at $\mathrm{pH} 7.3$ ) and $\left[{ }^{1} \mathrm{O}_{2}\right]_{\mathrm{ss}}$ of $2.4 \times 10^{-13} \mathrm{M}$ (under UVA light). These values were then used to estimate the fraction of ${ }^{1} \mathrm{O}_{2}$ reactivity in natural water. Using natural water from a pond in Switzerland and UVA light, a sample student found that cimetidine is mostly degraded by ${ }^{1} \mathrm{O}_{2}$ (73\%), while ranitidine degradation is controlled by direct photolysis $(62 \%)$, with only a minor contribution from ${ }^{1} \mathrm{O}_{2}$ (13\%). The remaining fractions (i.e., $27 \%$ and $25 \%$ for cimetidine and ranitidine, respectively) are due to reaction with other photochemically produced reactive intermediates.

\section{DISCUSSION}

This experiment succeeded in accomplishing the five major pedagogical goals established in the Introduction. First, by studying the reactivity of over-the-counter drugs in local natural water under natural or simulated sunlight, students readily appreciated the connection to their everyday lives. Learning that pharmaceuticals are designed to elicit targeted biological effects made it easy for them to understand the potential threat to aquatic ecosystems.

Next, the vast majority of students correctly concluded in their lab reports that ranitidine degraded primarily via direct photolysis while cimetidine degraded primarily via indirect photolysis by comparing the pseudo-first order degradation rate constants in phosphate buffer and natural water. When students acquired their own UV-vis spectra, they better understood how the overlap of the absorbance spectra with the solar irradiance spectrum related to the photolysis pathway compared with when the spectra were provided by the instructor.

With respect to the third pedagogical goal, nearly all of the students successfully generated natural logarithm versus time kinetic plots to confirm that the depletion kinetics was first- order with respect to ranitidine and cimetidine. A small number of students in the lower-division environmental chemistry course struggled with minor graphing issues, so care should be taken to support students who have limited experience with spreadsheet and graphing software. Students accurately used their kinetic plots to determine rate constants and to calculate half-lives and direct photolysis quantum yields. Most students struggled to understand the wavelength summation in the quantum yield calculations; a short tutorial with example data on this topic proved to be helpful. When the investigation was conducted remotely, the use of a spreadsheet template with embedded comments (see the Excel spreadsheet containing spectral and kinetic data sets) aided students in understanding the data analysis process. In general, students should be encouraged to pay special attention to the units for kinetic parameters.

Next, when students performed UV-vis spectroscopy and HPLC analyses, they generally did so effectively with verbal guidance from the instructor or teaching assistant. Students successfully integrated chromatograms and interpreted chromatographic peak areas as proportional to analyte concentration. In some semesters, students documented their HPLC analysis by writing a methods-style section of a scientific paper. By and large, students thoroughly described all of the relevant instrumental details.

Finally, students effectively used experimental controls and interpreted the resultant data to accomplish the primary experimental objectives, as reflected by their answers to prompted questions in the lab reports. By running dark controls, students readily deduced that all degradation in lightexposed samples was due to photolysis rather than other chemical degradation pathways. In the lower-division environmental chemistry course, students designed experiments with appropriate controls in consultation with the instructor to assess the impact of their chosen independent variable on photolysis rates.

When the basic modules were run together with advanced modules, student-instructor engagement and critical thinking were enhanced when student learning was assessed multiple times throughout the process rather than through a single final lab report. Providing scaffolded opportunities for students to demonstrate their learning motivated students to seek prompt support and feedback via email exchanges or online meetings, which was especially important when the investigation was conducted remotely.

In the remote learning context, allowing students to work through the investigation at their own pace by providing prerecorded introductory tutorials and comprehensive and detailed data analysis protocols proved to be more effective than explaining directly how calculations should be performed. The remote version of the lab provided some unique learning opportunities. Students had more time to focus on data analysis and interpretation, leading to a more critical analysis of their results than has been previously observed by the authors in an in-person laboratory context. Students also worked on a more individual basis in the remote learning context and were therefore more likely to ask more questions and to engage more deeply with the material.

\section{CONCLUSION}

The described experiment provided an authentic environmental context for students to learn about photochemistry and reaction kinetics while developing skills in instrumental 
analysis. The basic and advanced modules and add-on choices allow an instructor to tailor the experience to meet key learning objectives in a wide array of courses across the chemistry curriculum. The versatile design provides a meaningful learning experience in a traditional laboratory setting and in a remote learning environment. The laboratory experiment requires only inexpensive chemicals and basic laboratory glassware, supplies, and instrumentation available in most university chemistry and environmental engineering/science departments.

\section{ASSOCIATED CONTENT}

\section{SI Supporting Information}

The Supporting Information is available at https://pubs.acs.org/doi/10.1021/acs.jchemed.0c01398.

Chemical hazards and precautions (PDF, DOCX)

Instructor prep notes (PDF, DOCX)

Student instructions (PDF, DOCX)

Spectral and kinetic data sets (XLSX)

\section{AUTHOR INFORMATION}

\section{Corresponding Authors}

Jeffrey M. Buth - Department of Chemistry and Biochemistry, University of Mount Union, Alliance, Ohio 44601, United States; orcid.org/0000-0001-7128-4272;

Email: buthjm@mountunion.edu

Douglas E. Latch - Department of Chemistry, Seattle University, Seattle, Washington 98122, United States; Email: latchd@seattleu.edu

\section{Authors}

Rachele Ossola - Department of Environmental System Science, ETH Zurich, 8092 Zürich, Switzerland; (1) orcid.org/0000-0003-4648-5958

Sarah B. Partanen - Department of Environmental System Science, ETH Zurich, 8092 Zürich, Switzerland; (1) orcid.org/0000-0002-5719-8367

Kristopher McNeill - Department of Environmental System Science, ETH Zurich, 8092 Zürich, Switzerland; (1) orcid.org/0000-0002-2981-2227

William A. Arnold - Department of Civil, Environmental, and Geo- Engineering, University of Minnesota-Twin Cities, Minneapolis, Minnesota 55455, United States; 이이.org/ 0000-0003-0814-5469

Meghan O'Connor - Department of Civil, Environmental, and Geo- Engineering, University of Minnesota-Twin Cities, Minneapolis, Minnesota 55455, United States

Complete contact information is available at:

https://pubs.acs.org/10.1021/acs.jchemed.0c01398

\section{Notes}

The authors declare no competing financial interest.

\section{ACKNOWLEDGMENTS}

The initial experiments that form the basis of the experiment were supported by the National Institutes for Water Resources/USGS National Water Quality Competitive Grants Program (W.A.A. and K.M.). The formulation of the outreach protocol was supported by the U.S. National Science Foundation (CBET 0967163 to W.A.A. and K.M.), and the formulation for more advanced students was also supported by the U.S. National Science Foundation (CBET 1434313 and
1434148 to D.E.L. and W.A.A.) and the Swiss National Science Foundation (Grant 200020_188565 to R.O. and S.B.P.). Support for the writing of this article through a J. S. Braun/Braun Intertec Visiting Associate Professorship in the Department of Civil, Environmental, and Geo- Engineering at the University of Minnesota is gratefully acknowledged (J.M.B.). We thank Dr. Kristen J. Skogerboe and Stephanie Remke for implementing initial versions of this experiment and providing useful feedback.

\section{REFERENCES}

(1) Pilot, A.; Bulte, A. M. W. The Use of "Contexts" as a Challenge for the Chemistry Curriculum: Its Successes and the Need for Further Development and Understanding. Int. J. Sci. Educ. 2006, 28 (9), 1087-1112.

(2) Van Berkel, B.; De Vos, W.; Verdonk, A. H.; Pilot, A. Normal Science Education and Its Dangers: The Case of School Chemistry. Sci. Educ. 2000, 9 (1), 123-159.

(3) Wenzel, T. J.; Austin, R. N. Environmental Chemistry in the Undergraduate Laboratory. Environ. Sci. Technol. 2001, 35 (15), 326A-331A.

(4) Sevian, H.; Talanquer, V. Rethinking Chemistry: A Learning Progression on Chemical Thinking. Chem. Educ. Res. Pract. 2014, 15 (1), 10-23.

(5) Hawker, D. Kinetics of Carbaryl Hydrolysis: An Undergraduate Environmental Chemistry Laboratory. J. Chem. Educ. 2015, 92 (9), $1531-1535$

(6) Kirby, M. E.; Bullen, J. C.; Hanif, M. D.; Heiba, H. F.; Liu, F.; Northover, G. H. R.; Resongles, E.; Weiss, D. J. Determining the Effect of $\mathrm{pH}$ on Iron Oxidation Kinetics in Aquatic Environments: Exploring a Fundamental Chemical Reaction To Grasp the Significant Ecosystem Implications of Iron Bioavailability. J. Chem. Educ. 2020, 97 (1), 215-220.

(7) Buth, J. M. Ocean Acidification: Investigation and Presentation of the Effects of Elevated Carbon Dioxide Levels on Seawater Chemistry and Calcareous Organisms. J. Chem. Educ. 2016, 93 (4), $718-721$.

(8) Evans, A. L.; Messersmith, R. E.; Green, D. B.; Fritsch, J. M. Degradation of Environmental Contaminants with Water-Soluble Cobalt Catalysts: An Integrative Inorganic Chemistry Investigation. J. Chem. Educ. 2011, 88 (2), 204-208.

(9) Kolpin, D. W.; Furlong, E. T.; Meyer, M. T.; Thurman, E. M.; Zaugg, S. D.; Barber, L. B.; Buxton, H. T. Pharmaceuticals, Hormones, and Other Organic Wastewater Contaminants in U.S. Streams, 1999-2000: A National Reconnaissance. Environ. Sci. Technol. 2002, 36 (6), 1202-1211.

(10) Bottoni, P.; Caroli, S.; Caracciolo, A. B. Pharmaceuticals as Priority Water Contaminants. Toxicol. Environ. Chem. 2010, 92 (3), $549-565$.

(11) Santos, L. H. M. L. M.; Araújo, A. N.; Fachini, A.; Pena, A.; Delerue-Matos, C.; Montenegro, M. C. B. S. M. Ecotoxicological Aspects Related to the Presence of Pharmaceuticals in the Aquatic Environment. J. Hazard. Mater. 2010, 175 (1), 45-95.

(12) Liney, K. E.; Hagger, J. A.; Tyler, C. R.; Depledge, M. H.; Galloway, T. S.; Jobling, S. Health Effects in Fish of Long-Term Exposure to Effluents from Wastewater Treatment Works. Environ. Health Perspect. 2006, 114 (Suppl.1), 81-89.

(13) Benotti, M.; Trenholm, R.; Vanderford, B.; Holady, J.; Stanford, B.; Snyder, S. Pharmaceuticals and Endocrine Disrupting Compounds in US Drinking Water. Environ. Sci. Technol. 2009, 43, 597-603.

(14) Boreen, A. L.; Arnold, W. A.; McNeill, K. Photodegradation of Pharmaceuticals in the Aquatic Environment: A Review. Aquat. Sci. 2003, 65, 320-341.

(15) Remucal, C. K. The Role of Indirect Photochemical Degradation in the Environmental Fate of Pesticides: A Review. Environ. Sci. Process. Impacts 2014, 16 (4), 628-653. 
(16) Clemes, T. P.; Singmaster, K. A.; Wade, E. A. Photochemistry of Chloropicrin. A Physical Chemistry Project. J. Chem. Educ. 2000, 77 (7), 898

(17) Thompson, M. P.; Agger, J.; Wong, L. S. Paternò-Büchi Reaction as a Demonstration of Chemical Kinetics and Synthetic Photochemistry Using a Light Emitting Diode Apparatus. J. Chem. Educ. 2015, 92 (10), 1716-1720.

(18) Croisant, M.; Bretz, S. L.; Konkolewicz, D. Investigating Radical Reactivity and Structure-Property Relationships through Photopolymerization. J. Chem. Educ. 2019, 96 (2), 348-353.

(19) Garcia, S. E. Zantac Pulled from Shelves by Walgreens, Rite Aid and CVS over Carcinogen Fears. The New York Times, September 30, 2019.

(20) Latch, D. E.; Stender, B. L.; Packer, J. L.; Arnold, W. A.; McNeill, K. Photochemical Fate of Pharmaceuticals in the Environment: Cimetidine and Ranitidine. Environ. Sci. Technol. 2003, 37 (15), $3342-3350$

(21) Latch, D. E. Chapter 8 The Role of Singlet Oxygen in Surface Water Photochemistry. In Surface Water Photochemistry; Royal Society of Chemistry, 2016; pp 139-165. DOI: 10.1039/978178262215400139.

(22) Hiebert, S. M. Teaching Simple Experimental Design to Undergraduates: Do Your Students Understand the Basics? Adv. Physiol. Educ. 2007, 31 (1), 82-92.

(23) Willett, K. L.; Hites, R. A. Chemical Actinometry: Using oNitrobenzaldehyde to Measure Lamp Intensity in Photochemical Experiments. J. Chem. Educ. 2000, 77 (7), 900.

(24) Laszakovits, J. R.; Berg, S. M.; Anderson, B. G.; O’Brien, J. E.; Wammer, K. H.; Sharpless, C. M. p-Nitroanisole/Pyridine and $p$ Nitroacetophenone/Pyridine Actinometers Revisited: Quantum Yield in Comparison to Ferrioxalate. Environ. Sci. Technol. Lett. 2017, 4 (1), $11-14$.

(25) Apell, J. N.; McNeill, K. Updated and Validated Solar Irradiance Reference Spectra for Estimating Environmental Photodegradation Rates. Environ. Sci. Process. Impacts 2019, 21 (3), 427437.

(26) Ossola, R.; Schmitt, M.; Erickson, P. R.; McNeill, K. Furan Carboxamides as Model Compounds To Study the Competition between Two Modes of Indirect Photochemistry. Environ. Sci. Technol. 2019, 53 (16), 9594-9603.

(27) Meek, S. J.; Pitman, C. L.; Miller, A. J. M. Deducing Reaction Mechanism: A Guide for Students, Researchers, and Instructors. J. Chem. Educ. 2016, 93 (2), 275-286. 